\title{
Dégénérescence de motoneurones chez des souris transgéniques portant un mutant de la superoxyde dismutase humaine
}

En 1993, une découverte importante révélait que des mutations de la superoxyde dismutase (SOD) étaient à l'origine de formes familiales de sclérose latérale amyotrophique $(\mathrm{m} / \mathrm{s}$ $n^{\circ} 4$, vol. 9, p. 469 et $n^{\circ} 5$, vol. 9, p. 643). Cette découverte, encore récemment confirmée [1], posait une fois de plus la question du rôle de la SOD en physiopathologie. Un modèle animal était très souhaitable. Des deux méthodes possibles, invalidation et transgénèse, c'est cette dernière seule qui a été employée jusqu'à présent. Plusieurs essais antérieurs, entrepris dès $1987\left(\mathrm{~m} / \mathrm{s} n^{\circ} 2\right.$, vol. 4, p. 127), n'avaient provoqué chez les souris ayant reçu de la SOD humaine que des réactions minimes. Ce travail a été repris par une équipe américaine (14 auteurs, de Chicago, IL, et Duarte, CA) [2]. Le transgène utilisé était la SOD humaine ; l'identification du gène a été assurée par amplification de l'ADN et celle de la SOD elle-même par une méthode immunologique. Trois allèles de la SOD ont été préparés : le gène sauvage et deux mutants ; l'un, Ala-4-Val, dont l'activité est très diminuée ; l'autre, Gly-93-Ala, dont l'activité est normale, et la stabilité modérément abaissée. Toutes les lignées portant le gène normal, les mutants A4V et la plupart des lignées G93A n'ont présenté aucun trouble notable. En revanche, une des lignées G93A, celle qui avait reçu le plus grand nombre de copies, a manif esté des troubles neurologiques importants. Une faiblesse des membres postérieurs apparut vers 3 à 4 mois, évoluant en quelques semaines vers des paralysies mort. Les autres lignées ayant reçu une dose moindre du même mutant restent sous surveillance.

Dans les neurones moteurs de la moelle, surtout ventraux, une perte d'axones et une baisse de la choline acétyltransférase ont été mises en évidence. On trouve aussi une perte des axones myélinisés dans les nerfs intramusculaires, partiellement compensée par des bourgeonnements. Comment interpréter de tels résultats? On constate que deux facteurs sont nécessaires : une activité importante, et une mutation. Une activité élevée de la SOD humaine normale ne provoque pas de troubles. L'apport d'un transgène pathologique, et non inactivé, est impératif : $\mathrm{A} 4 \mathrm{~V}$, inactif ou peu actif, est sans effet. Comme il faut un nombre élevé de copies (18 par génome diploïde), on ne peut pas exclure un effet de position pour l'insertion de certaines de ces copies. Par ailleurs, il ne semble pas qu'il s'agisse d'un résultat de hasard non reproductible. Deux autres groupes auraient obtenu des résultats analogues, non encore publiés [3].

On se trouve ainsi confronté à une maladie neurologique provoquée, chez l'homme, par des mutations qui aboutissent à une diminution de l'activité, chez la souris, par une surcharge importante en une enzyme mutée. Cette SOD, enzyme énigmatique, chargée en principe de détoxifier les radicaux libres d'oxygène, mais dont les modes d'action sont loin d'être tous connus, a été dotée de bien des vertus. On la considère, en particulier, comme active contre la sénescence; si des transgènes de SOD ont, en effet, prolongé la durée de vie chez des drosophiles $\left(\mathrm{m} / \mathrm{s} n^{\circ} 5\right.$, vol. 10 , p. 610), il est à noter que les travaux sur les souris transgéniques ne font pas mention de tels résultats.

Pour expliquer les observations de Gurney et al. [2], il faut faire appel à une conception nouvelle : les atteintes neurologiques ne seraient pas dues à une insuffisance, ou à un excès, de l'activité propre de la SOD, mais à une altération qualitative qui conférerait à l'enzyme mutée des propriétés nouvelles, un "gain de fonction ", qui serait dominant. A l'appui de ce concept, l'observation que, dans le transgène actif comme dans la dizaine de mutations connues, chez l'homme, il s'agit de mutations faux-sens, qui ne suppriment pas la synthèse de la protéine. Reste à trouver la nature de l'action toxique. Restera aussi à comprendre comment une même maladie est liée, dans une partie des formes familiales, à des anomalies de la SOD, mais rarement dans les formes isolées de sclérose latérale amyotrophique, qui constituent pourtant la grande majorité des cas.

J.C.D.

1. Rosen DR, Bowling AC, Patterson D, et al. A frequent ala4 to val superoxide dismutase-1 mutation is associated with a rapidly progressive familial amyotrophic lateral sclerosis. Hum Mol Genet $1994 ; 3: 981-7$.

2. Gurney ME, Pu H, Chiu AY, et al. Motor neuron degeneration in mice that express a human $\mathrm{Cu}, \mathrm{Zn}$ superoxide dismutase mutation. Science $1994 ; 264: 1772-5$.

3. Marx J. Mouse model found for ALS. Science $1994 ; 264: 1663-4$ 